(1)

CrossMark

\title{
Intragraft donor-specific antibodies and lung transplantation
}

\author{
Jussi M. Tikkanen ${ }^{1}$ and Kathryn Tinckam ${ }^{2,3}$ \\ Affiliations: ${ }^{1}$ Toronto Lung Transplant Program, Toronto General Hospital, University Health Network, \\ Toronto, ON, Canada. ${ }^{2} \mathrm{HLA}$ Laboratory, Laboratory Medicine Program, Toronto General Hospital, University \\ Health Network, Toronto, ON, Canada. ${ }^{3}$ Division of Nephrology, Dept of Medicine, Toronto General Hospital, \\ University Health Network, Toronto, ON, Canada.
}

Correspondence: Jussi M. Tikkanen, University Health Network, 200 Elizabeth Street, M5G2C4, Toronto, ON, Canada. E-mail: jussi.tikkanenđuhn.ca

@ERSpublications

Intragraft DSA can be found in explanted lungs of patients with chronic lung allograft dysfunction in the absence of circulating DSA. New diagnostic approaches to assess for intragraft DSA may add to our diagnostic arsenal in treating AMR. http://bit.ly/31kS00b

Cite this article as: Tikkanen JM, Tinckam K. Intragraft donor-specific antibodies and lung transplantation. Eur Respir J 2019; 54: 1901937 [https://doi.org/10.1183/13993003.01937-2019].

A lung transplant recipient has suddenly developed new circulating donor-specific antibodies (DSAs). Are these antibodies causing antibody-mediated allograft injury or are they merely a marker of immune activation? Should one treat the patients with no allograft dysfunction? Are some DSAs more important than others? Yet another patient appears to have chronic lung allograft dysfunction (CLAD) and yet no history of circulating DSAs. In these increasingly recognised scenarios, how should the transplant physician respond?

DSA development after lung transplantation carries a higher risk of CLAD [1-5]; however, not all patients with DSA develop any harmful sequelae and many patients clear them from their circulation regardless of therapeutic approach [6]. Also, the diagnosis of antibody-mediated rejection in lung transplant recipients is difficult as both histopathology and C4d staining are non-specific.

In this issue of the European Respiratory Journal, SACREAS et al. [7] present important new intragraft DSA data from explanted lung allografts in patients who had developed severe CLAD. The authors clearly demonstrate the presence of intragraft DSAs in rejecting lungs and particularly in cases where circulating DSAs are no longer detected.

The authors used the same single-antigen bead (SAB) assay as VISENTin et al. [8] in their earlier report. As the authors clearly state, this approach has not been validated, but the choice of a positive threshold, although arbitrary (as in most SAB assays), is mathematically defined and used consistently. As such, this approach appears valid and as a result of several washing steps, the intragraft antibodies detected are unlikely due to sample contamination by serum containing circulating antibodies. This is supported by the fact that in many cases, no circulating antibodies were observed even if intragraft DSAs were present. No human leukocyte antigen (HLA) antibodies that were non-donor specific were observed in the grafts, further supporting the notion that the DSAs in the allograft were true intragraft DSAs.

The study included 36 explanted lungs from CLAD patients: 18 with bronchiolitis obliterans syndrome and 18 with restrictive allograft syndrome (RAS). Out of these, 11 patients (31\%) had developed serum DSAs at some time during their follow-up, consistent with the published literature [4-6]. In most cases, 
the specificity of the intragraft DSAs reflected that of serum DSAs, and the majority of DSAs were HLA-DQ-DSA. This is in line with the findings of Visentin et al. [8] who showed that 10 out of 14 patients who had intragraft DSAs in transbronchial biopsy specimens had HLA-DQ-DSA and, in general, the serum mean fluorescence intensity of the DSAs was higher in patients who also had intragraft DSAs. Also, RAS was more common in the intragraft DSA group, reinforcing the concept that DSAs may contribute to RAS. Therefore, the results of this study align with other observations that the type of DSA matters and that HLA-DQ-DSA seems to be the most deleterious form [5, 9-11].

Notably, in six patients, circulating DSAs had cleared, but the explant nonetheless showed continued intragraft DSAs. This is interesting as there is some earlier evidence from the same group suggesting that clearance of DSAs is associated with better survival compared to persistence of DSAs [12]. Several explanations for this finding are possible. Firstly, the DSAs seen in this study developed generally later (3-5 years postoperatively) than in other studies, in which DSAs developed in the first year $[5,6]$. It is possible that the late DSAs have a different pathophysiology compared to early DSAs, where clearance rates of $>50 \%$ have been observed [6]. However, it may also be that some DSAs are more likely to bind to the allograft than others, or bind for longer than others, again supporting the notion that all DSAs are likely not equal. However, it is difficult to draw firm conclusions from the individual patient data, even considered in aggregate, given small total numbers.

The specificity of HLA antibodies reported in general may warrant a more detailed analysis and description than that which was discussed in this manuscript. Though conventionally reported as antigen level specificities, we must emphasise that antibody specificity is in fact biologically driven at the epitope or eplet level [13] rather than a whole antigen. Table 2 from their study suggests this was considered by the authors in those patients where more than two DSAs are identified at a given locus (e.g. DQ7/8/9 identified in three patients). Donors may only have up to two antigens at any locus; it is likely that in these cases the authors are correctly identifying antibody to epitope(s) known to be shared between DQ7, 8 and 9 antigens (specifically the beta chains of these antigens coded by DQB1 ${ }^{\star} 03$ ). A more detailed analysis of not only donor-specific antigens, but also donor-specific epitope directed antibodies (that may be identified through an analysis of non-DSA "third party" HLA antibodies also detected in serum), may further illuminate the dynamic interplay between serum and graft antibodies and better characterise those patients who appear, at the antigen level, to be serum-negative while graft-positive. In our centre it is common to see, in either the presence or absence of DSAs, the presence of third-party antibodies that also share epitopes with the conventionally identified antigen level DSA. It will be important in further iterations of this important work to include data regarding third party antibodies and their corresponding contributory epitopes, supporting greater understanding of the nature of antibody identification, circulation and binding.

Indeed the observed differences between serum and intragraft DSAs may be the most important observation of this study, given the treatment and prognosis implications for patients who may demonstrate one but not the other. In its current format in explanted lungs, the intragraft DSA assay cannot be used in prospectively applied clinical practice. However, if this test evolved into a reliable and subsequently validated assay to assess for intragraft DSAs from transbronchial biopsies, a comparable diagnostic assay could be of added value, where lung function still remains good in the presence of serum DSAs, or conversely where lung function is declining or pathology is suggestive of rejection, in the absence of serum DSAs. This could help us better tailor the immunosuppressive therapies we give our patients. The dynamic nature of antibody production and consumption on the endothelium is difficult to quantify in amount or timing, and antibody identification requires more attention to epitope specificities than antigens; it is therefore unsurprising that serum DSAs alone cannot detect or perfectly discriminate between clinically relevant HLA antibodies and apparent bystanders. While the present assay is not yet prime-time ready, the results of this study level set the requirements for more nuanced diagnostic and prognostic approaches in understanding antibody-mediated rejection, and highlight the importance of delving directly into the allograft in our diagnostic strategies beyond traditional pathology.

Conflict of interest: None declared.

\section{References}

1 Brugiere O, Suberbielle C, Thabut G, et al. Lung transplantation in patients with pretransplantation donor-specific antibodies detected by luminex assay. Transplantation 2013; 95: 761-765.

2 Morrell RM, Pilewski JM, Gries CJ, et al. De novo donor-specific HLA antibodies are associated with early and high-grade bronchiolitis obliterans syndrome and death after lung transplantation. J Heart Lung Transplant 2014; 33: $1288-1294$

3 Safavi S, Robinson DR, Soresi S, et al. De novo donor HLA-specific antibodies predict development of bronchiolitis obliterans syndrome after lung transplantation. J Heart Lung Transplant 2014; 33: 1273-1281. 
4 Snyder LD, Wang Z, Chen DF, et al. Implications for human leukocyte antigen antibodies after lung transplantation: a 10-year experience in 441 patients. Chest 2013; 144: 226-233.

5 Tikkanen JM, Singer LG, Kim SJ, et al. De novo DQ donor-specific antibodies are associated with chronic lung allograft dysfunction after lung transplantation. Am J Respir Crit Care Med 2016; 194: 596-606.

6 Hachem RR, Yusen RD, Meyers BF, et al. Anti-human leukocyte antigen antibodies and preemptive antibody-directed therapy after lung transplantation. J Heart Lung Transplant 2010; 29: 973-980.

7 Sacreas A, Taupin J-L, Emonds M-P, et al. Intragraft donor-specific anti-HLA antibodies in phenotypes of chronic lung allograft dysfunction. Eur Respir J 2019; 54: 1900847.

8 Visentin J, Chartier A, Massara L, et al. Lung intragraft donor-specific antibodies as a risk factor for graft loss. J Heart Lung Transplant 2016; 35: 1418-1426.

9 Roux A, Bendib Le Lan I, Holifanjaniaina S, et al. Antibody-mediated rejection in lung transplantation: clinical outcomes and donor-specific antibody characteristics. Am J Transplant 2016; 16: 1216-1228.

10 Walton DC, Cantwell L, Hiho S, et al. HLA class II eplet mismatch predicts de novo DSA formation post lung transplant. Transpl Immunol 2018; 51: 73-75.

11 Walton DC, Hiho SJ, Cantwell LS, et al. HLA matching at the eplet level protects against chronic lung allograft dysfunction. Am J Transplant 2016; 16: 2695-2703.

12 Verleden SE, Vanaudenaerde BM, Emonds MP, et al. Donor-specific and -nonspecific HLA antibodies and outcome post lung transplantation. Eur Respir J 2017; 50: 1701248.

13 Tambur AR, Claas FH. HLA epitopes as viewed by antibodies: what is it all about? Am J Transplant 2015; 15: $1148-1154$. 